\title{
Avaliação de programas e profissionalização da gestão pública
}

Jeni Vaitsman ${ }^{1}$

Rômulo Paes-Sousa²

1 Doutora em Sociologia pelo Instituto Universitário de Pesquisas do Rio de Janeiro (luperj). Pesquisadora-titular da Escola Nacional de Saúde Pública, da Fundação Oswaldo Cruz. Foi Diretora de Avaliação e Monitoramento do Ministério do Desenvolvimento Social e Combate à Fome (2004 - 2007).

2 Médico, PhD em Epidemiologia pela London School of Hygiene and Tropical Medicine (University of London). Pesquisador do Observatório de Iniquidades em Saúde, da Fundação Oswaldo Cruz. Foi Secretário de Avaliação e Gestão da Informação do Ministério do Desenvolvimento Social e Combate à Fome (2004 - 2007). Desde abril de 2010 é o Secretário Executivo do MDS. 


\title{
Resumo
}

O PRESENTE ARTIGO PROCURA DESTACAR ALGUNS ELEMENTOS

DECISIVOS PARA O SUCESSO E INSTITUCIONALIZAÇÃO DO MODELO

DE SISTEMA DE AVALIAÇÃO E MONITORAMENTO DESENVOLVIDO NO

Ministério do Desenvolvimento Social e Combate À Fome.

SÃO DESTACADAS A ARTICULAÇÃO, A COOPERAÇÃO E A PARTICIPA-

ÇÃO DAS SECRETARIAS E DEPARTA-

MENTOS ENVOLVIDOS NA CONSTRU-

ÇÃO DO REFERIDO MODELO E COMO

A MOBILIZAÇÃO E A CONFLUÊNCIA DE

INTERESSES EM RELAÇÃO A GANHOS

COMUNS VIABILIZARAM A SUA INSTI-

TUCIONALIZAÇÃO.

\begin{abstract}
THIS ARTICLE ATTEMPTS TO HIGHLIGHT SOME KEY ELEMENTS FOR THE SUCCESS AND THE INSTITUTIONALIZATION OF THE EVALUATION AND MONITORING SYSTEM MODEL DEVELOPED BY THE MINISTRY OF SOCIAL DEVELOPMENT AND FIGHT AgAINST Hunger. THE TEXT COVERS ASPECTS SUCH AS ARTICULATION, COOPERATION AND PARTICIPATION OF THE SECRETARIATS AND DEPARTMENTS INVOLVED IN CONSTRUCTING THE SYSTEM AND HOW THE MOBILIZATION AND THE CONFLUENCE OF INTERESTS IN JOINT GAINS MADE POSSIBLE ITS INSTITUTIONALIZATION.
\end{abstract}

\section{PALAVRAS-CHAVE:}

Programas sociais; Políticas públicas; Avaliação; Monitoramento; Gestão pública

Revista Brasileira de Monitoramento e Avaliação | Número 1 | Janeiro-Junho de 2011 


\section{Introdução}

No Brasil, a expansão dos sistemas de avaliação integrados às estruturas governamentais vem produzindo algumas mudanças no âmbito da gestão das políticas públicas. Com alguma tradição nas áreas da educação e da saúde, mais recentemente, a avaliação tem sido institucionalizada na área de proteção social não contributiva, conquistando cada vez maior legitimidade como atividade mais integrada ao processo das políticas. Este trabalho é um relato do caso do Ministério do Desenvolvimento Social e Combate à Fome (MDS), criado em 2004 e que, desde então, constrói um sistema de avaliação e monitoramento por meio de uma unidade específica para este fim. Se, por um lado, a introdução desses mecanismos significa maior profissionalização da gestão, por outro, não se trata de um processo sem os tradicionais conflitos entre as esferas técnica e a política.

Os autores do trabalho, que participaram da formulação e implementação do sistema, fazem uma reflexão sobre o período de 2004 a 2007, quando a área foi implementada e contratou ou codesenvolveu 72 estudos de avaliação dos seus, então, 22 programas.

A construção de um sistema de avaliação e monitoramento dos programas e políticas do MDS significou a implementação de uma política de avaliação e monitoramento, o que dependeu de um conjunto de elementos de natureza institucional e política. Em um Ministério novo, formado pela junção de órgãos federais já existentes anteriormente, com culturas profissionais e áreas de atuação distintas, os problemas enfrentados podem ser entendidos como problemas de implementação de uma inovação dentro de organizações.

Nesta reflexão ex-post, descrevemos o modelo do sistema de avaliação e monitoramento desenvolvido no MDS procurando destacar alguns elementos que consideramos como decisivos para seu sucesso e institucionalização. Entre esses elementos, mostramos como a cooperação pressuposta no modelo e a participação das secretarias e departamentos envolvidas possibilitaram a mobilização e a confluência de interesses em relação a ganhos comuns que viabilizam sua institucionalização.

Na primeira seção, fazemos uma breve discussão sobre a dualidade político-gerencial que caracteriza o campo da avaliação de políticas e programas; na segunda seção, discutimos os mecanismos de cooperação e confiança, que se colocam como centrais para o enfrentamento de desafios organizacionais; na terceira seção, descrevemos o processo de institucionalização do sistema de avaliação no Ministério do Desenvolvimento Social e Combate à Fome durante os três primeiros anos de sua existência, de 2004 a 2006, e as características de seu modelo. Finalmente, fazemos algumas considerações finais sobre o sistema de avaliação construído e seu papel para a profissionalização da gestão pública. 
Avaliação,

\section{gestão e política}

A discussão sobre os limites, significados e papéis atribuídos à avaliação de políticas e programas por diferentes vertentes teóricas e disciplinares é extensa. Move-se desde uma visão gerencialista que considera a avaliação como parte dos mecanismos de accountability desenvolvidos nas sociedades contemporâneas e que contribuiria com a efetividade e transparência das ações governamentais até os que ressaltam muito mais sua dimensão política e uso em função de interesses políticos (MOKATE, 2003; CAIDEN, G.E. \& CAIDEN, N., 2001; GÉVA-MAY, 1999; GREENE，1994; OSZLAK, 2005; FARIA, 2005; GUBA, E. \& LINCOLN, Y., 1989).

Sem entrar nessa discussão, nossa perspectiva é entender o processo de construção do sistema de avaliação do MDS a partir da dualidade de uma área que é ao mesmo tempo técnica e política. Ou seja, trata-se de reconhecer as características específicas da avaliação de políticas e programas públicos, uma atividade exercida em um terreno atravessado pela política, onde os estudos extrapolam o simples âmbito da produção de conhecimento. Ainda que realizados dentro dos parâmetros científicos socialmente reconhecidos, enquanto pesquisa aplicada às políticas públicas, acabam fazendo parte das disputas políticas do setor avaliado ou mesmo da política de um modo geral. Não se trata, portanto, de um conhecimento teórico descontextualizado, mas de um tipo de conhecimento que pode produzir alta carga política sobre programas ou valores em disputa (PATTON, 1987; WEISS, 1987; GREENE, 1994).

Nas sociedades democráticas contemporâneas, em que atores governamentais e não governamentais participam e disputam políticas, os resultados dos estudos acabam tendo efeitos políticos, principalmente quando revelam aspectos não esperados ou contrários aos objetivos que haviam sido previstos para o programa avaliado. Resultados, quando divulgados, podem ser usados tanto para tomadas de decisões por parte dos gestores contratantes da avaliação, quanto por outros atores envolvidos na disputa por determinada agenda política. Uma vez que atores e interesses existem dentro e fora das estruturas governamentais, a divulgação de resultados de avaliação, ainda que internamente, também pode assumir uma conotação política. A divulgação externa de resultados sempre repercute sobre gestores, dirigentes e a comunidade de políticas envolvidos nos programas avaliados.

A confluência entre produção de conhecimento e política, típica do campo da avaliação, estreita-se quando o que está em jogo são programas com grande visibilidade política e social e, sobretudo, quando a legitimidade e a eficácia desses programas são contestadas por atores com alto poder de vocalização e formação de agenda. Por outro lado, a própria

Revista Brasileira de Monitoramento e Avaliação | Número 1 | Janeiro-Junho de 2011 
existência da avaliação como mecanismo institucionalizado produz não apenas mudanças de atitudes em relação aos mecanismos de melhoria da ação governamental, mas induz também um debate político mais bem informado sobre políticas e programas. Se a avaliação como instrumento de gestão pode ser capaz de produzir maior clareza e debate público sobre as políticas, somente a política poderá produzir transformações nas políticas.

Com todos os conflitos políticos que possam envolver os resultados de estudos de avaliação, é inegável seu papel de produzir accountability. Embora esse termo seja muitas vezes traduzido como responsabilização, Oszlak (2005) observa que ele se refere a um processo de prestação de contas; no entanto, o termo accountability conota a própria obrigação de se prestar contas, assumida voluntariamente pelo sujeito, sem necessidade da intervenção de um terceiro para exigi-la. Essa distinção é crucial, pois a obrigação faz parte dos valores e, portanto, da cultura; não precisando, necessariamente, ser exigida por outros. A menção ao termo accountability evocaria, de imediato, relação com as noções de transparência, eficiência, eficácia, bom governo, autonomia, controle, serviço ao cidadão, legitimidade, ou, inclusive, democracia. E também pareceria associada a seus opostos: arbitrariedade, opacidade, corrupção, ineficiência, autoritarismo etc.

Ainda que a necessidade de mecanismos de controle e accountability seja fortemente ressaltada pelas vertentes gerencialistas, essa não é uma problemática restrita à esfera administrativa ou de gestão e que deveria ser cativa de uma única visão teórica. Tais mecanismos respondem à pressão da sociedade por maior transparência e responsabilização da gestão pública, como parte do Estado democrático: "distintas correntes de pensamento hoje reconhecem que a sorte da democracia e, de certo modo, do bom governo, está inextricavelmente unida à possibilidade de instaurar efetivos mecanismos de responsabilização da função pública" (OSZLAK, 2005, p. 248).

A institucionalização de diferentes mecanismos de accountability, entre os quais a avaliação, tornou-se parte da governança nos Estados democráticos e onde existem diferentes mecanismos de prestação de contas. No Brasil, essa institucionalização é parte do processo mais amplo de construção da democracia e da profissionalização da administração pública, em que um conjunto de controles de diferentes naturezas foi se estabelecendo a partir da Constituição de 1988.

A avaliação pode agregar qualidade à política pública em um campo estratégico onde se disputam questões de legitimação política e social (SUBIRATS, 2005) e constituir um dos mecanismos que contribuem para diminuir aquilo que Cunill-Grau identifica como um "déficit de controle da sociedade sobre o Estado" (CUNILL-GRAU, 2004, p. 44). Dentro de uma visão que associa a melhoria do controle da administração pública à ampliação da democracia, a construção da função pública profissional constitui uma das condições básicas para a democratização do sistema político, em que o funcionalismo profissional teria forte incidência sobre a capacidade do estado em contribuir com certo grau de autonomia à formulação de políticas. 
Por outro lado, do ponto de vista da melhoria da capacidade de gestão do Estado, a execução de estudos de avaliação só adquire relevância se esses e seus resultados fizerem parte de sistemas de avaliação, o que remete ao problema da institucionalização desses sistemas no interior de estruturas governamentais. Decerto, esse processo depende de um conjunto de condições, entre as quais a decisão política de implantar um sistema de avaliação, com a consequente alocação de recursos financeiros, físicos e humanos. Depende também da cooperação entre os membros da organização.

\section{Cooperação e confiança}

Em qualquer organização, o sucesso de uma inovação, como a criação e institucionalização de uma estrutura de avaliação, exige legitimidade e adesão dos membros da organização, o que pressupõe, entre outras coisas, que eles acreditem na relevância daquilo que estão implementando, ou seja, nas práticas de avaliação.

Como qualquer inovação, a implementação do sistema de avaliação no MDS exigiu a criação de uma base comum de entendimento para a atuação conjunta entre as secretarias responsáveis pela condução dos programas e a secretaria responsável pela avaliação. Ou seja, foi preciso conseguir resolver dois problemas básicos de qualquer organização social: cooperação e confiança.
A cooperação é vista por vários autores como um dos principais problemas da implementação. Uma vez que as pessoas em diferentes cargos, posições e autoridade têm que trabalhar juntas, seja no interior de uma organização com vários departamentos, ou entre várias organizações, quando elas precisam cooperar, isso é facilitado quando há interdependência de recursos, congruência de objetivos e confiança mútua (CLINE, 2000; LUNDIN, 2007). Por outro lado, a confiança, uma das bases para a ação coletiva e a produção de bens públicos, é apontada como central na capacidade das organizações em administrar sua dinâmica de modo eficiente e assegurar sua sobrevivência e crescimento, em contextos que envolvem a coordenação de vários tomadores de decisão (COLEMAN, 1999; PUTNAM, 1996; KLIKSBERG, 1999; TYLER, 2003; OWEN \& VIDERAS, 2008).

No caso do MDS, confiança e cooperação foram tão indispensáveis quanto a necessidade, para a atividade de avaliação, de compartilhar bancos de dados gerenciais sobre os programas, como cadastro de beneficiários, dados físico-financeiros, além de informação, conhecimento sobre os programas e contatos com municípios. A cooperação intraorganizacional entre diferentes unidades responsáveis pela gestão da política, no plano executivo, e pela avaliação foi imprescindível para implementação da avaliação.

Revista Brasileira de Monitoramento e Avaliação | Número 1 | Janeiro-Junho de 2011 


\section{O modelo criado no MDS}

O modelo representa a institucionalização de uma atividade no interior desta unidade do Governo Federal, ainda que seja efetuada por pesquisadores contratados externamente e estritamente orientada para responder perguntas referentes ao planejamento, implementação ou repercussões de programas públicos. O termo programa é utilizado como denominação geral de uma política, programa ou ação governamental.

A criação do MDS reuniu distintas trajetórias organizacionais, campos de conhecimento e objetos de atuação, com suas próprias visões de mundo, modos de agir e soluções para a condução de suas atividades e resolução de problemas. Não existia, em nenhuma das organizações anteriores que se fundiram no novo ministério, cultura de avaliação, ou seja, um conjunto de práticas e crenças incorporando a avaliação como parte da gestão de programas e políticas. Pelo contrário, havia grande desconhecimento do significado e da própria função avaliação.

As bases normativas da institucionalização da avaliação no MDS foram estabelecidas com a criação do próprio Ministério em janeiro de $2004^{3}$ e com a definição de sua estrutura organizacional em maio do mesmo ano. 0 MDS fundiu o Ministério da Assistência Social (MAS), o Ministério Extraordinário de Segurança Alimentar e Combate à Fome (MESA) e a Secretaria-Executiva do Programa Bolsa Família, a qual era vinculada diretamente à Presidência da República.
O novo Ministério começou então a responder pelas políticas nacionais de desenvolvimento social, de segurança alimentar e nutricional, de assistência social e de renda de cidadania, que ficaram a cargo, respectivamente, das secretarias "finalísticas" que se originaram dos antigos órgãos: Secretaria Nacional de Segurança Alimentar (SESAN); Secretaria Nacional de Assistência Social (SNAS); Secretaria Nacional de Renda de Cidadania (SENARC). Foram também criadas duas secretarias para o desenvolvimento de atividades que vieram a ser chamadas de "meio": a Secretaria de Articulação Institucional e Parcerias (SAIP) e a Secretaria de Avaliação e Gestão da Informação (SAGI). Essas duas secretarias apresentam, na estrutura do MDS, a mesma posição que as secretarias finalísticas, são unidades que se relacionam horizontalmente na hierarquia organizacional; acima delas encontra-se somente a Secretaria-Executiva, além, é claro, do Ministro.

Coube à SAGI as funções de avaliação e monitoramento das políticas e programas de desenvolvimento social do MDS. Isso significou uma inovação em termos de gestão pública brasileira, uma vez que até então não existia, em nenhum Ministério, uma secretaria com essa finalidade exclusiva ${ }^{4}$. Do ponto de vista regimental, as competências da SAGI foram formuladas pelas instâncias dirigentes do MDS, no processo de sua criação. Contudo, nesse momento, isso não significou a formulação, tampouco a implementação; apenas a instituição de suas bases normativas. 
Nesse âmbito, cabe diferenciar atividades de controle e de avaliação, que, às vezes, são confundidas entre si. Enquanto o foco das atividades de avaliação e monitoramento é a qualidade da gestão, o das atividades de controle é a legalidade dos atos da administração pública, o cumprimento das normas e dos procedimentos legais.

O objetivo mais geral do sistema de avaliação consistiu na produção de informação baseada em evidências e sistematizada sobre programas, políticas, ações, serviços; na produção de insumos para mudanças nos programas que pudessem melhorar seu desempenho não apenas nas metas previstas, mas também em seus objetivos. Do ponto de vista gerencial, enquanto função incorporada à rotina da execução das políticas públicas, seu objetivo foi o de subsidiar tomadas de decisões e ações de um conjunto de atores interessados.

Avaliações de programas podem ser feitas por meio de diferentes tipos de estudos, que observam várias de suas dimensões, tais como: relevância, eficiência, efetividade, resultados e impactos. Espera-se que as indicações desses estudos contribuam para melhorar a qualidade dos processos de implementação ou verificar os resultados dos programas, dando também subsídios para o planejamento, a programação e a tomada de decisões futuras.

O risco de contaminação pelos interesses políticos de organizações, grupos ou indivíduos está na raiz da recomendação de que as avaliações sejam executadas por instituições externas. Em nossa experiência, houve uma razão suplementar a essa recomendação: a possibilidade de se agregar aos estudos maior diversidade e maior volume de expertise na observação de vários aspectos de um programa. A constituição de uma equipe interna à organização capaz de cobrir os vários campos de saber para a avaliação de programas tende a ser de alto custo, de limitada capacidade de produção de estudos de qualidade e de maior risco de endogenia nas escolhas metodológicas.

Em uma estrutura de avaliação cuja rationale é a legitimidade científica, esta é também a base para legitimidade política, interna e externamente. A qualidade técnica dos estudos tornouse um capital político, subsidiando o debate público sobre os principais programas da área.

As escolhas dos desenhos de estudos de avaliação devem ser vistas também como parte desse ambiente, onde os métodos dos estudos dependem das perguntas que se quer responder, dos recursos e das fontes de da-

3 Medida Provisória n. 163, de 23 de janeiro de 2004, transformada na Lei n. 10.868, de 13 de maio de 2004.

4 As atribuições da SAGI, apresentadas no Anexo I, foram definidas pelo Decreto n. 5.074, de 11 de maio de 2004 , e corroboradas pelo Decreto n. 5.550, de 22 de setembro de 2005 .

Revista Brasileira de Monitoramento e Avaliação | Número 1 | Janeiro-Junho de 2011 
dos disponíveis e do tempo de resposta desejado. Os parâmetros adotados pela SAGI, no MDS, seguiram a tipologia clássica da área quanto às dimensões que podem abordadas pelos estudos de avaliação (DRAIBE, 2001):

A) PROCESSOS - estudos sobre os pontos que favorecem ou dificultam os processos de implementação da política ou do programa, incluídos seus desenhos, suas dimensões organizacionais e institucionais. Os resultados das avaliações de processos podem ser mais facilmente utilizados por gestores e gerentes, que têm a possibilidade de tomar providências em relação a achados e recomendações pertinentes.

B) RESULTADOS - englobam: desempenho, que se refere aos produtos definidos pelas metas do programa; impacto, que indica mudança na situação dos beneficiários, provocada diretamente pelo programa. As avaliações de impacto procuram comparar as respostas dadas por dois ou mais grupos similares da população quanto às condições de exposto e não exposto a um determinado programa. Há ainda o componente efeitos, que se refere aos resultados sociais ou institucionais não esperados de um programa.

Os estudos de impacto, geralmente considerados o "padrão-ouro" da avaliação, usam métodos quantitativos de coleta de dados e modelos estatísticos/econométricos para analisá-los; baseiam-se em estratégias amostrais complexas e em desenhos quaseexperimentais. Essa configuração possui alta legitimidade na comunidade internacional de avaliação e produz informações mensuráveis sobre a demanda e a oferta das políticas públicas. No entanto, os estudos requerem recursos financeiros consideráveis e expertise nem sempre disponível, além de exigir tempo demorado para obter resultados. Os principais usuários dos estudos mais robustos de impacto são tomadores de decisão e formuladores de programas nacionais ou transnacionais, gestores de níveis hierárquicos mais altos e pesquisadores envolvidos com o tema.

As avaliações de processos ou de desempenho, por sua vez, são de realização mais factível, porque são mais flexíveis metodologicamente. São geralmente aplicadas em estudos de processos ou de desempenho, podendo ser realizadas mais rapidamente.

Quanto maiores ou mais complexos forem a abrangência temática, a representatividade da população observada, o grau de aprofundamento das questões, a combinação de métodos, o número de estratos de uma amostra e a quantidade de cruzamentos pretendida na análise, mais demorada e cara será uma pesquisa. Em compensação, uma pesquisa mais robusta metodologicamente pode ter maior peso na definição dos rumos de determinados programas, desde que não ultrapasse o tempo máximo esperado para a produção das respostas às perguntas formuladas. O tempo da produção de conhecimento não costuma coincidir com o tempo da política e isso é um problema inerente à dualidade da área, que sempre se tenta antecipar, mas como qualquer política implementada, nem sempre de acordo com o previsto. De qualquer forma, não impediu a institucionalização do sistema. 


\section{O processo de institucionalização}

O MDS desenvolveu um modelo com algumas singularidades: posicionamento horizontal na estrutura organizacional; concentração de recursos humanos com alta qualificação; contratação externa dos estudos de avaliação; ênfase na gestão dos contratos de pesquisa e disseminação dos resultados; desenvolvimento de instância ministerial de pactuação da agenda de avaliação e financiamento com recursos oriundos, em grande parte, de empréstimos junto às agências internacionais.

Essa iniciativa significou uma inovação na gestão pública brasileira, uma vez que, até então, não existia, em nenhum Ministério, uma secretaria com essa finalidade exclusiva. Comparativamente às demais experiências latino-americanas, a posição dessa unidade na estrutura organizacional do Ministério também é singular. Em países como Chile, Argentina e México, a função avaliação ora está concentrada num órgão gestor do planejamento de todo o sistema governamental (caso chileno), ora tem suas funções distribuídas em várias unidades de gestão e controle no interior dos Ministérios de Desenvolvimento Social (casos argentino e mexicano).

Essa posição na estrutura organizacional, sua autonomia relativa dentro de um órgão recente, reunindo secretarias com diferentes trajetórias, precisava ser legitimada. Como organizações são locais de conflito, dificilmente, resistências a processos inovadores são resolvidas apenas por procedimentos hierárquicos, sem a criação de mecanismos de cooperação e construção de confiança. A participação dos membros das secretarias finalísticas na elaboração da agenda e na implementação da avaliação - definição do desenho e das questões de pesquisa, construção de instrumentos de pesquisa, discussão de relatórios e recomendações, incorporação das recomendações pertinentes - garantiu a adesão e legitimidade ao processo, criando as bases para sua sustentabilidade, a despeito de subsequentes mudanças nos cargos de direção.

O convencimento dos membros da organização e, sobretudo, dos gestores de que as informações produzidas por monitoramento e avaliação possibilitariam não apenas melhorar o desempenho dos programas, mas também verificar se os resultados previstos estavam sendo alcançados, assim como possíveis reformulações, consistiu em uma etapa necessária do processo de institucionalização.

A legitimidade da avaliação passa pelo reconhecimento de sua utilidade. Enquanto atores racionais, os gestores de programas têm interesse pela função instrumental da avaliação e é isso que dá sentido ao investimento em estudos acadêmicos. A cooperação entre diferentes membros do corpo técnico, pesquisadores externos e gestores dos programas consistiu em grande incentivo para a cooperação.

Revista Brasileira de Monitoramento e Avaliação | Número 1 | Janeiro-Junho de 2011 
Com a centralização da função avaliação na SAGI, a unidade tornou-se responsável por realizar ou contratar todos os estudos de avaliação referentes aos programas sob gestão do Ministério. Também ficou a cargo da capacitação dos gestores do MDS em atividades de avaliação, com o objetivo de fornecer-lhes maior competência para definir suas demandas; debater as metodologias escolhidas e os resultados encontrados e, principalmente, apropriar-se dos resultados das investigações para a melhoria dos programas e políticas sob sua gestão.

Ainda que do ponto de vista regimental as competências da SAGI tenham sido formuladas pelas instâncias dirigentes do Ministério à época de sua criação, isso não implicou a formulação, tampouco a implementação, de uma política ou um modelo de avaliação; apenas a instituição de suas bases normativas. Crucial para a implementação de qualquer empreendimento é a capacidade institucional da agência responsável por isso. 0 primeiro efeito da criação de uma unidade responsável por avaliação foi a construção de capacidade institucional, envolvendo recursos financeiros, físicos e humanos. Além da alocação dos recursos próprios, os oriundos de empréstimos de organismos internacionais foram fatores indutores da implementação e institucionalização do processo.

A exigência de critérios técnicos e profissionais para desempenhar um conjunto de atividades de avaliação - definição de estudos, desenhos e metodologias; contratação; acompanhamento e avaliação dos estudos contratados - requer recursos humanos qualificados. A inexistência de um corpo de funcionários próprio, capacitado para essas atividades, consistiu uma debilidade enfrentada de diferentes formas, inicialmente por meio de contratos precários. A partir de 2007, as contratações precárias foram substituídas por mecanismos mais estáveis.

Para avaliar os programas das três grandes áreas do Ministério, investiu-se em competência técnica e formação multidisciplinar, recrutando-se profissionais oriundos de diferentes áreas de atuação e de conhecimento: ciências sociais e humanas, tecnologia da informação, demografia, estatística, economia, nutrição, assistência social, agronomia etc. 0 objetivo foi constituir uma unidade compacta, com a maior competência técnica agregada possível (Tabela 1).

O financiamento externo e o consequente relacionamento com agências internacionais contribuíram para aumentar a qualidade do debate interno sobre o papel da avaliação na gestão pública. A relação com atores externos reforçou a necessidade de produção de evidências científicas, fortalecendo os atributos de independência e transparência buscados nos estudos e seus resultados. É aqui que a qualidade técnica e científica dos estudos de avaliação pode cumprir não apenas as clássicas funções gerenciais, mas também a de legitimidade política de sua função no âmbito de uma gestão pública profissionalizada. 
- tabela 1 - RECursos humanos da secretaria de AVALIAÇÃo e GESTÃO DA INFORMAÇÃO SEGUNDO FUNÇÃO E TITULAÇÃO MÁXIMA - JULHO DE 2006

\begin{tabular}{|c|c|c|c|c|c|c|}
\hline \multirow[b]{2}{*}{ Função } & \multicolumn{4}{|c|}{ TITULAÇÃO MÁXIMA } & \multirow[b]{2}{*}{ Doutorado } & \multirow[b]{2}{*}{ Total } \\
\hline & $\begin{array}{l}\text { Ensino } \\
\text { Médio }\end{array}$ & Graduação & Especialização & Mestrado & & \\
\hline Direção & & 2 & 1 & 3 & 5 & 11 \\
\hline Administração/Gestão & & 1 & 2 & & & 3 \\
\hline Apoio Administrativo & 2 & 3 & & & & 5 \\
\hline Técnico & 3 & 13 & 1 & 9 & 2 & 28 \\
\hline Estagiários Técnicos & 3 & & & & & 3 \\
\hline Total & $8^{* x}$ & $19 *$ & 4 & 12 & 7 & 50 \\
\hline
\end{tabular}

Fonte: SAGI/MDS

No ano de 2006, foi constituída a política de avaliação e monitoramento do MDS (BRASIL, 2006), que define como ações de avaliação os estudos e pesquisas com os seguintes objetivos:

a) análise da implementação de programas;

b) análise de resultados imediatos dos programas;

c) análise de impactos ou efeitos dos programas;

d) análise da eficiência, da equidade, da eficácia e da efetividade de programas;

e) análise do perfil dos beneficiários dos programas; f) elaboração de diagnósticos de perfil da demanda;

g) avaliação da satisfação de beneficiários/usuários;

h) avaliação da qualidade dos serviços prestados;

i) elaboração de estudos de "linha de base".

Revista Brasileira de Monitoramento e Avaliação | Número 1 | Janeiro-Junho de 2011 
A agenda de avaliação passou a ser regida pelo Plano Anual de Monitoramento e Avaliação elaborado por grupo de trabalho com representação dos atores relevantes envolvidos na gestão dos programas, sob coordenação da SAGI. Aprovado, anualmente, no mês de agosto, o Plano indica os programas e ações do MDS a serem priorizados pelas avaliações no ano seguinte (VAITSMAN; RODRIGUES; PAES-SOUSA, 2006). A elaboração do Plano considera as avaliações realizadas anteriormente pelo MDS ou por outras instituições, de modo a evitar repetições e desperdício de recursos.

\section{O fluxo de avaliação dos programas do MDS}

Quem demanda e utiliza os resultados dos estudos de avaliação? No caso do MDS, demandas sobre questões e temas a serem avaliados podiam partir de dirigentes, gestores e gerentes de diferentes níveis. A divulgação de resultados de avaliações atinge a comunidade de políticas envolvidas com o tema da proteção social, públicos com interesses variados, tendo, portanto, distintas implicações. Há sempre diversos tipos de audiência para os resultados da avaliação: gestores e técnicos envolvidos com os programas avaliados, outros gestores públicos, pesquisadores, jornalistas e demais interessados nas áreas acadêmica e política.

De modo geral, o fluxo de avaliação dos programas do MDS pode ser dividido em: 1 ) definição da demanda, 2) contratação dos estudos e gestão dos contratos e 3) disseminação dos resultados. A definição da demanda com- preende as etapas da discussão e preparação dos termos de referência. O Termo de Referência (TR) é utilizado para estabelecer os parâmetros técnicos e jurídico-administrativos necessários aos editais de seleção e contratação de pessoa física ou jurídica para a execução das pesquisas. Os parâmetros técnicos definem escopo, objetivos, metodologia, cronograma e custos do projeto.

Os parâmetros jurídico-administrativos referem-se aos requisitos exigidos pelas normas. Entre estes últimos, enfatizamos dois aspectos: a propriedade dos microdados por parte do MDS e a adequação técnica da equipe executora.

Uma inovação no ciclo da avaliação do modelo desenvolvido no MDS foi a disponibilização dos microdados das pesquisas por meio do Consórcio de Informações Sociais (CIS), preservando-se a confidencialidade dos indivíduos observados. A publicação dos microdados torna mais eficiente a utilização dos recursos públicos investidos em pesquisa e promove a transparência em relação aos resultados desses investimentos. Permite a confrontação e comparação dos resultados divulgados com resultados oriundos de outras análises, promovendo uma validação informal das informações divulgadas sobre os programas. Do ponto de vista político, a divulgação pública é um mecanismo que aumenta o controle da sociedade sobre o governo. Aqui também, a boa gestão implica maior accountability e transparência. A seguir, apresentamos, de maneira esquemática, o fluxo de avaliação dos programas sociais do MDS. 


\section{FLUXO DE AVALIAÇÃO DOS PROGRAMAS DO MDS}
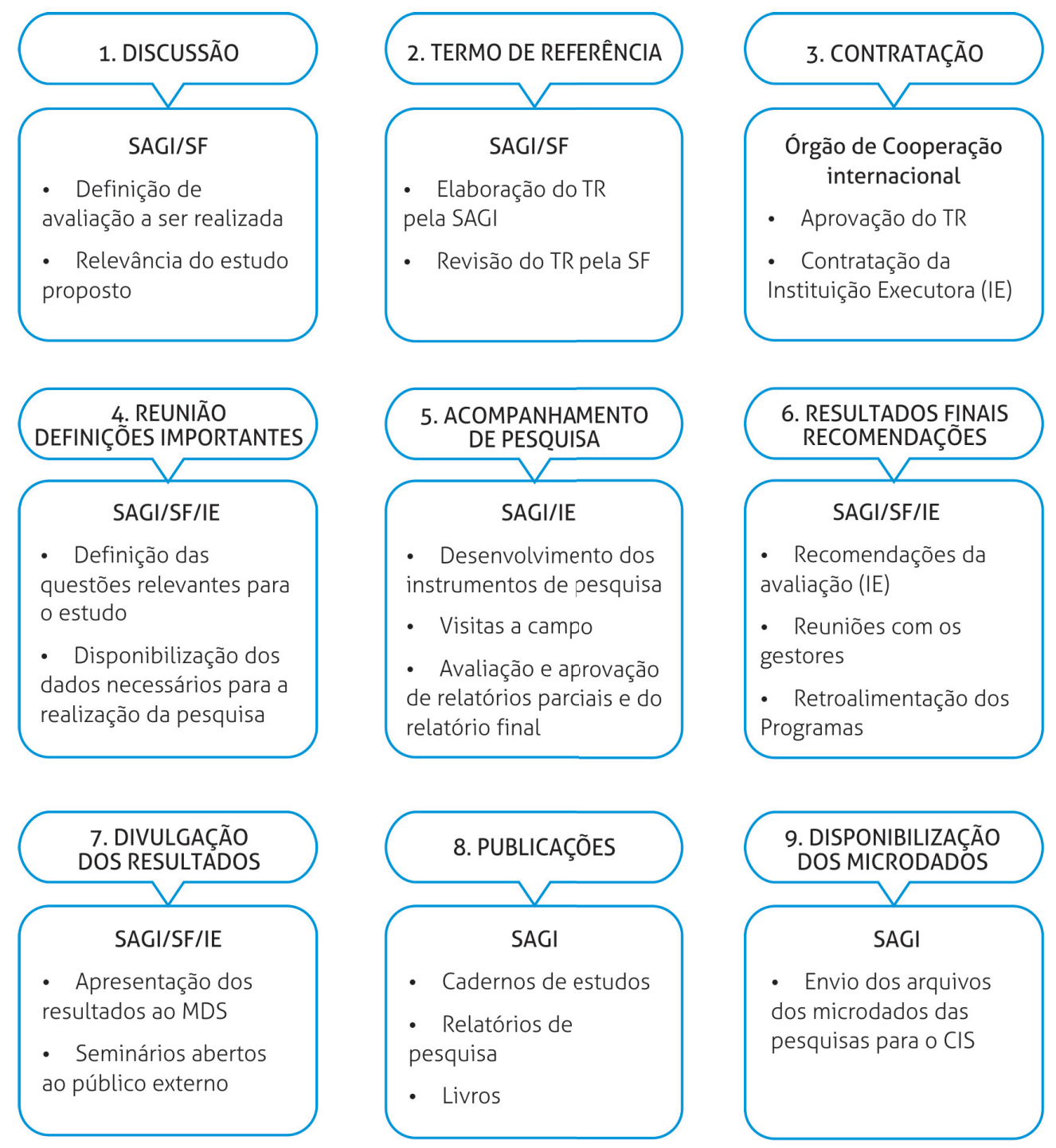

- SAGI = Secretaria de Avaliação e Gestão da Informação

- $\mathrm{SF}=$ Secretaria Finalística

- $\quad \mathrm{IE}=$ Instituição Executora

- $\operatorname{MDS}=$ Ministério do Desenvolvimento Social e Combate á Fome

- $\mathrm{CIS}=$ Consórcio de Informações Sociais

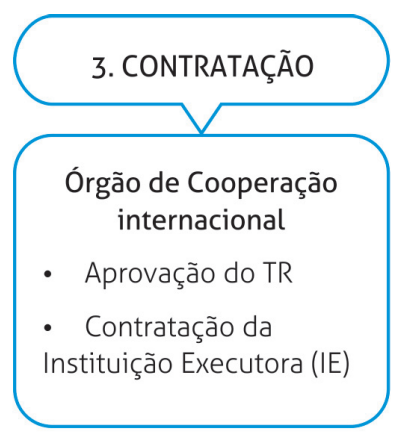

Revista Brasileira de Monitoramento e Avaliação | Número 1 | Janeiro-Junho de 2011 


\section{Considerações finais}

A experiência do MDS mostra que, na perspectiva da institucionalização e legitimidade, é difícil separar a função de gestão da avaliação de suas funções estratégicas. Pois, para os gestores, é justamente sua utilidade enquanto instrumento de gestão que fomenta a cooperação e, ao mesmo tempo, legitima a agenda da avaliação, promovendo maior participação dentro da organização. Processos mais transparentes e cooperativos fomentam a adesão ao processo de accountability e criam condições para a incorporação, pelos membros da organização, da cultura da avaliação.

As contratações externas, garantindo maior independência e isenção dos estudos, constituíram elementos decisivos para a construção de legitimidade interna e externa. Do ponto de vista interno, o envio permanente dos resultados das investigações às instâncias de pactuação renova a autoridade dos avaliadores perante os avaliados, reduzindo os potenciais conflitos entre estes.

A utilização dos resultados de avaliação pelas diferentes audiências depende do grau de domínio e interesse sobre os programas e políticas, bem como do conhecimento das metodologias de avaliação. A divulgação, por meio de publicações de resultados de avaliação, além de tornar público os produtos das políticas e programas, subsidia o debate técnico e político da área de desenvolvimento social.

Nesse sentido, a apropriação e o uso dos resultados da investigação pela gestão das políticas e programas, que de fato, coroariam o ciclo de avaliação de um programa ou política, constituem um desafio ao mesmo tempo político e gerencial. Questões como a não coincidência entre o tempo da política e o da produção de conhecimento costumam ser objeto de conflitos entre os diferentes atores envolvidos no processo de implementação e avaliação. Muitas vezes, recomendações relevantes de estudos de boa qualidade não conseguem interferir na dinâmica das políticas e programas, seja pela indiferença dos gestores ou dificuldade de mobilizar os responsáveis por mudanças nessa dinâmica.

A experiência do MDS é muito recente, mas pode ser inserida no processo de profissionalização da administração pública brasileira (ABRÚCIO, 2007; NOGUEIRA \& CAVALCANTI, 
2009). Os estudos de avaliação realizados cumpriram seus objetivos de produção de evidências em políticas públicas. Ganhos de informação, análises e subsídios para a expansão e o planejamento dos programas foram conquistas obtidas à base de cooperação organizacional e legitimidade interna e externa, pautadas por critérios de transparência e excelência. A avaliação conseguiu um lugar institucional, passando não apenas a ser aceita, mas fortemente demandada como necessária pelas demais unidades e membros da organização.

As atividades da unidade de avaliação desempenharam tanto funções estratégicas, de governo, ao promoverem a transparência das ações do Estado, quanto operacionais, ao produzirem evidências para os gestores de programas. Ainda que o sistema tenha sido implementado a partir de um modelo top-down, dentro de uma perspectiva gerencialista, a cooperação entre os atores envolvidos garantiu a participação de vários níveis hierárquicos da organização. A dinâmica do processo de contratação de estudos e produção e divulgação de resultados constituiu-se em elemento importante para a construção de uma gestão mais eficaz e transparente.

Ainda que a institucionalização de sistemas de avaliação integrados às estruturas governamentais dependa de inúmeros fatores sobre os quais nem sempre seus formuladores ou implementadores têm poder de decisão e influência, a experiência do MDS, ao instituir um sistema de avaliação, expandiu o debate qualificado sobre os projetos e políticas de governo. A discussão de questões que são objeto das políticas, com base em evidências, produz opiniões e julgamentos mais informados, promovendo mudanças de qualidade na arena das políticas públicas.

Com todas as dificuldades que a implementação de sistemas de avaliação e monitoramento apresenta, seja quanto à sua legitimidade organizacional, seja quanto ao uso de seus resultados, a avaliação, enquanto uma atividade integrada às estruturas governamentais, não apenas produz evidências que podem contribuir para melhorar a eficácia dos programas e políticas públicas, mas também contribui com o processo de construção de uma gestão pública mais transparente e profissionalizada.

Revista Brasileira de Monitoramento e Avaliação | Número 1 | Janeiro-Junho de 2011 


\section{Referências bibliográficas}

ABRUCIO, Fernando Luiz. Trajetória recente da gestão pública brasileira: um balanço crítico e a renovação da agenda de reformas. Revista Brasileira de Administração Pública, Rio de Janeiro: FGV, p. 6786. 2007. Edição Especial Comemorativa.

BRASIL. Ministério do Desenvolvimento Social e Combate à Fome. Portaria sobre a política de avaliação e monitoramento do MDS. Brasília, DF: MDS, 2006.

CAIDEN, G. E; CAIDEN, N. Enfoques y lineamiento, la mediciona y la evaluation des desenpeño en programas del sector publico. Revista do Serviço Público, v. 52, n. 1, p. 78-103, 2001.

CLINE, Kurt D. Defining the implementation problem: organization management versus cooperation. Journal of Public Administration Research and Theory, Oxford, v. 10, n. 3, 2000.

COLEMAN, James. Social capital in the creation of human capital. In: DAS GUPTA, Partha; SERAGELDIN, Ismail. Social capital: a multifaceted perspective. Washington, DC: The World Bank, 1999.

CUNILL-GRAU, Nuria. La Democratización de la administración pública: los mitos a vencer. In: POLÍTICA y gestión pública. Buenos Aires: Centro Latinoamericano de Administración para el Desarrollo; Fondo de Cultura Económica de Argentina, S.A., 2004.
DRAIBE, S. M. Introdução. In: BARREIRA, M. C. R. N.; CARVALHO, M. do C. B. de (Orgs.). Tendências e Perspectivas na Avaliação de Políticas Sociais. São Paulo:IEE/PUC-SP, 2001.

FARIA, Carlos Aurélio P. A Política da avaliação de políticas. Revista Brasileira de Ciências Sociais, São Paulo, v. 20, n. 59, 2005.

GEVA-MAY, I; PAL, L. Good Fences Make Good Neighbors - Policy Evaluation and Policy Analysis - Exploring the Differences, Evaluation, v. 5, n.3, p.259-277. 1999.

GUBA, E. G. \& LINCOLN, Y. S. Fourth Generation Evaluation. Newbury Park, CA, Sage Publications, 1989.

GREENE, Jennifer C. Qualitative Program Evaluation: practice and promise. In: DENZIN, N; Lincoln. Y. Handbook of Qualitative Research. London: Sage Publications, 1994. p.530-544.

LUNDIN, Martin. Explaining cooperation: how resource interdependence, goal congruence, and trust affect joint actions in policy implementation, J Public Adm Res Theory, v.17, n. 4, p. 651-672. 2007.

KLIKSBERG, Bernardo. Capital social y cultura, claves esenciales del desarrollo. Revista de la Cepal, Santiago de Chile, n. 69, dez. 1999. 
MOKATE, K. Convirtiendo el "monstruo" en aliado: la evaluación como herramienta de la gerencia social. Washington, D. C.: Banco Interamericano de Desenvolvimento, 2003.

NOGUEIRA, Ronaldo; CAVALCANTE, Pedro. Avaliação e monitoramento: duas experiências de institucionalização na gestão das políticas públicas no Poder Executivo Federal Brasileiro. In: CONGRESO INTERNACIONAL DEL CLAD SOBRE LA REFORMA DEL ESTADO Y DE LA ADMINISTRACIÓN PÚBLICA, 14, 2009, Salvador (Bahia), Brasil.

OSZLAK, Oscar. Responsabilización o respondibilidad?: el sujeto y el objeto de un Estado responsable. In: RESPONSABILIZACION y Evaluación de la gestión pública. Caracas: Centro Latinoamericano de Administración para El Desarrollo; Editorial Texto, C.A., 2005. p. 239-272.

OWEN, Ann L; VIDERAS, Julio. Trust, cooperation, and implementation of sustainability programs: the case of Local Agenda 21, Ecological Economics, Elsevier, v. 68 , n. 1-2, p. 259-272, December 2008.

PATTON. Evaluations's political inherency: practical implications for design and use. In: PALUMBO,D. J. The Politics of program evaluation. Newbury Park, CA. Sage, 1987. p. 100-145.
PUTNAM, Robert D. Comunidade e democracia: a experiência da Itália moderna. Rio de Janeiro: Fundação Getúlio Vargas, 1996.

SUBIRATS, Joan. Podemos utilizar los instrumentos de evaluación como palanca de gobierno del sector público? In: RESPONSABILIZACIÓN y Evaluación de la gestión pública. Caracas: Centro Latinoamericano de Administración para el Desarrollo ; Editorial Texto, C.A., 2005. p.47-58.

TYLER. Trust within organisations, Personnel Review, v. 32 , n. 5, p.556-568. 2005.

VAITSMAN, Jeni; RODRIGUES, Roberto W. S; PAES-SOUSA, Rômulo. O Sistema de avaliação e monitoramento das políticas e programas sociais: a experiência do Ministério do Desenvolvimento Social e Combate à Fome. Brasília: MDS; UNESCO, 2006. Policy Papers, n.17.

WEISS, C. Where politics and evaluation research meet. In: Palumbo, D. J. (Ed.) The politics of program evaluation. Newbury Park, CA. Sage Publications, 1987. 